\title{
Drug-resistant Headache and Cranio-facial Pain: Rationale for Greater Occipital Nerve Stimulation
}

\author{
Lavano Angelo*, Della Torre Attilio, Valente Vinicio, Lavano Francesco and Guzzi Giusy
}

Department of Neurosurgery, University Hospital of Germaneto, Campus "Salvatore Venuta", Catanzaro, Italy

\section{Introduction}

Despite the advances in the management of headache disorders, many patients suffering from chronic migraine are refractory to current treatments. New treatments backed by good evidence are therefore in great need. Electrical stimulation of peripheral nerves is used to treat various painful conditions including headache disorders. More recently it has been reported good outcome in these painful conditions with subcutaneous placement of leads at the level of $\mathrm{C} 1$ and stimulation of the greater occipital nerve: This procedure is named Occipital Nerve Stimulation (ONS) [1-5]. The clinical conditions treated with ONS include occipital neuralgia, Trigeminal Autonomic Cephalalgias (TACs), migraine and transformed migraine.

The surgical procedure is divided in two steps. In the first step, with the patient in prone position under local anaesthesia, a vertical incision of $2 \mathrm{~cm}$ is made in posterior cervical region in the midline on the external occipital protuberance. Under fluoroscopy control, a Tuohy needle is passed transversely in the subcutaneous space across the base of the greater occipital nerve at the level of $\mathrm{C} 1$ starting from the midline incision towards the mastoid process and following the trajectory of the nuchal line. A quadripolar wire lead is introduced into the Touhy needle by medial to lateral direction; under fluoroscopy control the needle is withdrawn and the lead is connected to an external cable for intraoperative testing with an external stimulator. The stimulation parameters are chosen to obtain paraesthesias in the innervation territory of the greater occipital nerve. The lead is then sutured to the underlying fascia with a silicone fastener and a silk sutures. In some cases, according to Franzini lead fixation techniques [6]. Pisces Quad lead may be fixed to the lateral portion of the superficial fascia transfixing the plastic tip of the lead with a suture needle through a vertical incision of $4 \mathrm{~cm}$ lateral to the external occipital protuberance. After a 7-15 days trial period of external stimulation, if the patient reported at least $50 \%$ decrease in headache intensity and/or frequency associated with a decrease headache medication use, a permanent implant of a single channel IPG or a dual-channel IPG is then performed under general anesthesia. The parameters used for chronic stimulation are usually: Frequency 30-60 Hz, Pulse Width 90-400 microsec, Amplitude 1.5-9.0 Volts, bipolar stimulation configuration, cyclic mode.

Weiner and Reed first described a percutaneous approach for Occipital Nerve Stimulation (ONS) in presumed occipital neuralgia [7]. Greater occipital nerve electrical stimulation could have effects on pain both in the area directly innervated by this nerve and in regions innervated by the trigeminal nerve. The greater occipital nerve is a medial branch of the posterior ramus of $\mathrm{C} 2$ spinal root and lies in the subcutaneous tissue. C2 dorsal horn converges with trigeminal nerve in the "trigemino-cervical complex" that is a neuronal column extending from trigeminal nucleus caudalis up to C3 level [8].

Convergence of trigeminal nerve and occipital nerve in the "trigemino-cervical complex" may explain how nociception activation at either end of this structure may result in both anterior and posterior pain: thus occipital nerve stimulation could have effects on pain not only in regions directly innervate by the occipital nerve but also in the areas innervated by the trigeminal nerve. The exact mechanism by which ONS produces analgesia is still incompletely understood: electrical stimulation of occipital nerve has both peripheral and central nervous system effects that may modulate nociception. Stimulation could give raise to depolarization blockade of the small diameter afferent fibers $A \delta$ and C. Alternately stimulation of the large diameter $A \beta$ afferent fibers may have an antinociceptive effect due to suppression of activity in $\mathrm{C}$ fibers and $A \delta$ fibers at the level of the spinal dorsal horn (gate control theory). ONS may influence supraspinal structures involved in central nociceptive trafficking, such the dorsal pons. PET study demonstrated, as a result of electrical stimulation of occipital nerve, an activation of this region that may be involved in descending pathways that control pain via stimulation of the trigemino-vascular system at the level of upper cervical pain $[3,8]$. Finally ONS may influence the pulvinar nucleus of the thalamus and the anterior cingulate cortex that are involved in the affective dimension of pain [9].

Use of ONS is reported in occipital neuralgia, cervicogenic headache, migraine, transformed migraine and also in trigeminal autonomic cephalalgias (TACs). TACs is a group of primary headache disorders recognised in the $2^{\text {th }}$ edition of the International Headache Society classification [10]. It is characterized by unilateral trigeminal distribution pain that occurs in association with prominent ipsilateral cranial autonomic features. The group comprises: Chronic Cluster Headache (CCH), Chronic Paroxysmal Headache (CPH), Continuous Headache $(\mathrm{CH})$ and Short-lasting Unilateral Neuralgiform headache attacks with Conjunctival injection and Tearing (SUNCT syndrome).

Deep Brain Stimulation of posterior hypothalamus is showed to be effective in TACs, mainly in Chronic Cluster Headache and SUNCT [11]. Anyway because of possible cerebral hemorrhagic complications (1-2\%), hypothalamic implantation should be used with caution. ONS in refractory chronic migraine patients was studied in 2 multi-centre randomized trials. In PRISM trial 125 drug-refractory chronic migraine patients were treated with ONS or sham, without any significant improvement [12]. In ONSTIM trial 39\% of patients treated with active ONS during 3 months had at least $50 \%$ reduction in headache frequency and/or a 3-point intensity scale decrease, while there was no improvement in the non-stimulated or ineffectively stimulated groups

*Corresponding author: Lavano Angelo, Department of Neurosurgery, University Hospital of Germaneto, Campus "Salvatore Venuta", Viale Europa, Catanzaro 88100, Italy, Tel: +39 09613647389; Fax: +39 09613647092; E-mail: lavano@unicz.it

Received March 25, 2016; Accepted March 26, 2016; Published March 28, 2016

Citation: Angelo L, Attilio DT, Vinicio V, Francesco L, Giusy G (2016) Drugresistant Headache and Cranio-facial Pain: Rationale for Greater Occipital Nerve Stimulation. J Pain Manage Med 2: e103.

Copyright: $\odot 2016$ Angelo L, et al. This is an open-access article distributed under the terms of the Creative Commons Attribution License, which permits unrestricted use, distribution, and reproduction in any medium, provided the original author and source are credited. 
[13]. Lead migration/dislodgement is the common complication due to the wide range of neck movements and the distance from the anchoring area to the IPG pocket [2]. Leads and technique of implantation evolved over time to minimize lead displacements: The transfixtion by a needle of the plastic tip of the Pisces Quad lead and the suturing of it to the superficial fascia with a non-absorbable suture proposed by Franzini has proven to be effective in preventing this complication [6]. Reported infection rates range from $4 \%$ to $30 \%$ with varied length of follow-up [14]. A case of subcutaneous tissue change at implant site and another case of reduction or loss of musculoskeletal control were reported in Silberstein et al. [15].

ONS may require an early replacement of the IPG because of power loss/battery failure. This is due to the high voltage needed to effectively stimulate the nerve which is not in anatomical contact with the lead; besides some type of TACs, as chronic cluster headache, need higher amplitude of stimulation for obtain benefits.

\section{Conclusions}

Subcutaneous Occipital Nerve Stimulation offers significantly effective prospect to attend patients suffering from severe headaches and cranio-facial pain syndromes resistant to medications and others conservative therapies. ONS is well a tolerated and safe procedure if compared with other invasive modality of treatment and with Deep Brain Stimulation. Further development of ONS and other similar techniques and validation of their effectiveness require both continuous improvement of clinical evidence and further studies on migraine pathophysiology and its responses to various forms of neuromodulation. Meanwhile, individual preferences of the patient and improvement in the evidence base by RCT can guide the application of ONS.

\section{References}

1. Vallejo R, Benyamin R, Kramer J (2006) Neuromodulation of the occipital nerve in pain management. Techniques in Regional Anestesia \& Pain Management 10: 12-15.
2. Schwedt TJ (2008) Occipital nerve stimulation for medically intractable headache. Curr Pain Headache Rep 12: 62-66.

3. Paemeleire K, Bartsch T (2010) Occipital nerve stimulation for headache disorders. Neurotherapeutics 7: 213-219.

4. Goadsby PJ (2007) Neuromodulatory approaches to the treatment of trigeminal autonomic cephalalgias. Acta Neurochir Suppl 97: 99-110.

5. Weiner RL (2007) Occipital neurostimulation for treatment of intractable headache syndromes. Acta Neurochir Suppl 97: 129-133.

6. Franzini A, Messina G, Leone M, Broggi G (2009) Occipital nerve stimulation (ONS). Surgical technique and prevention of late electrode migration. Acta Neurochir (Wien) 151: 861-865.

7. Weiner RL, Reed KL (1999) Peripheral neurostimulation for control of intractable occipital neuralgia. Neuromodulation 2: 217-221.

8. Lee P, Huh BK (2013) Peripheral nerve stimulation for the treatment of primary headache. Curr Pain Headache Rep 17: 319.

9. Lavano A, De Rose M, Guzzi G, Romano M, Della Torre A, et al. (2015) Neurostimulation for the treatment of Cluster Headache. Austin J Anesthesia and Analgesia 3: 1041

10. de Tommaso M, Shevel E, Pecoraro C, Sardaro M, Losito L, et al. (2006) Topographic analysis of laser evoked potentials in chronic tension-type headache: correlations with clinical features. Int J Psychophysiol 62: 38-45.

11. Broggi G, Franzini A, Leone M, Bussone G (2007) Update on neurosurgical treatment of chronic trigeminal autonomic cephalalgias and atypical facial pain with deep brain stimulation of posterior hypothalamus: results and comments. Neurol Sci 28: 138-145.

12. Lipton R, Goadsby P, Cady R (2009) PRISM study: occipital nerve stimulation for treatment-refractory migraine. Echolalia pp: 29-30.

13. Saper JR, Dodick DW, Silberstein SD, McCarville S, Sun M, et al. (2011) Occipital nerve stimulation for the treatment of intractable chronic migraine headache: ONSTIM feasibility study. Cephalalgia 31: 271-285.

14. Chen YF, Bramley G, Unwin G, Hanu-Cernat D, Dretzke J, et al. (2015) Occipital nerve stimulation for chronic migraine--a systematic review and metaanalysis. PLoS One 10: e0116786.

15. Silberstein SD, Dodick DW, Saper J, Huh B, Slavin KV, et al. (2012) Safety and efficacy of peripheral nerve stimulation of the occipital nerves for the management of chronic migraine: results from a randomized, multicenter double-blinded, controlled study. Cephalalgia 32: 1165-1179. 